\title{
MARS: Parallelism based Metrically Accurate 3D Reconstruction System in Real-Time
}

\author{
Shu Zhang ${ }^{1}$, Ting Wang ${ }^{2}$, Gongfa $\mathrm{Li}^{3}$, Junyu Dong ${ }^{*}, 1$, and Hui $\mathrm{Yu}^{*}{ }^{*} 4$
}

\begin{abstract}
-
Due to the increasing application demands, lightweight device-based 3D recovery draws many attentions from a wide group of researchers in both academic and industrial fields. The current 3D reconstruction solutions are commonly achieved either using depth data or RGB data. The depth data usually come from a deliberately designed hardware for specific tasks, while the RGB data-based solutions only employ a single RGB camera with vision-based computing algorithms. Limitations are expected from both. Depth sensors are commonly either bulky or relatively expensive compared to RGB cameras, thus of less flexibility. Normal RGB cameras usually have better mobility but less accuracy in 3D sensing than depth sensors. Recently, machine learning based depth estimation has also been presented. However, its accuracy are still limited. To improve the flexibility of the 3D reconstruction system without loss in accuracy, this paper presents a solution of unconstrained Metrically Accurate 3D Reconstruction System (MARS) for 3D sensing based on a consumer-grade camera. With a simple initialization from a depth map, the system can achieve incremental 3D reconstruction with a stable metric scale. Experiments are conducted using both real-world data and public datasets. Competitive results are obtained using the proposed system compared with several existing methods.
\end{abstract}

Index Terms-3D reconstruction, metrical, outlier removal, random down sampling

\section{INTRODUCTION}

$\mathrm{T}$ HE perception of the depth has received wide attentions in many fields in recent years [1], such as 3D visual odometry [2], virtual reality and smart community [3], [4]. Various kinds of depth sensors have been developed for different applications. For example, Microsoft's Xbox utilizes indoor depth data obtained by Kinect for gamerconsole interactions; Google's robotic cars utilize detailed 3D maps of their surroundings obtained from multiple sensors such as laser rangers to achieve autonomous driving. Currently, existing approaches either use depth sensors or RGB cameras for 3D data acquisition. In general, depth sensor based methods rely on a dedicated hardware to obtain 3D information. This hardware utilizes techniques such as Infrared Structure Light, Stereo Vision, Time-ofFlight (TOF), and Photometric Stereo. Therefore, compared to a mobile RGB camera, these techniques make the depth sensing system bulkier and less mobile in most scenarios, for example with a large baseline. This can pose limitations for the practical applications of these depth sensing systems for mobile device. Moreover, if the depth sensing hardware is made as compact as an RGB camera for a better mobility,

${ }^{1}$ Ocean University of China, Qingdao, China.

2 Shandong University of Science and Technology, Qingdao, China.

${ }^{3}$ Wuhan University of Science and Technology, Wuhan, China.

${ }^{4}$ University of Portsmouth, Portsmouth, UK.

* Correspondence Authors: Junyu dong (dongjunyu@ouc.edu.cn) and Hui Yu (hui.yu@port.ac.uk)

This work was supported by the Engineering and Physical Sciences Research Council (EPSRC) (EP/N025849/1); National Natural Science Foundation of China (NSFC) (41906177, 41927805, 51575407); China Postdoctroal Science Fondation Grant (2019M652476); the Fundamental Research Funds for the Central Universities, China (201964022); International Science and Technology Cooperation Program of China (ISTCP) (2014DFA10410). the system is likely to have an increased price, though the prices of both the depth sensor and the RGB camera have been dropping in recent years. Moreover, the performance of the depth sensor is also limited by certain environments such as outdoors or underwater. For example, Kinect is only designed for indoor applications. The data transition of the sensors can also present potential concerns when achieving fast mobile computing $[5 \mid-[8]$.

To avoid these problems, many researchers tend to employ single RGB camera based methods with vision-based algorithms such as Simultaneous Localization and Mapping (SLAM) and Structure from Motion (SFM) for 3D reconstruction. RGB images are most intuitive data form delievering a detailed environment information [9]. They are also much easier to be captured with cheaper imaging device. These methods only utilize a single RGB camera, which reduces equipment costs and provides better mobility. For instance, a cheap RGB camera can be easily mounted on a mobile drone for collision avoidance through 3D information sensation. In contrast, only compact wireless depth sensors could do the same job, which are usually expensive and still less common in the consumer-grade market. However, in practice, existing RGB camera based methods still have limitations. For example, SLAM only focuses on ego pose estimation rather than $3 \mathrm{D}$ reconstruction. In most cases, it also requires a detectable reference object as a bootstrap for system initialization $\mid 10]$, or another specified initialization process such as a certain motion of the camera |11|. SFM systems are designed for offline 3D reconstruction due to its global optimization. It requires to input all images at the same time to find the best feature matching results for system initialization. Moreover, it is also hard to generate metrically scaled 3D structures. Recently, machine learning 


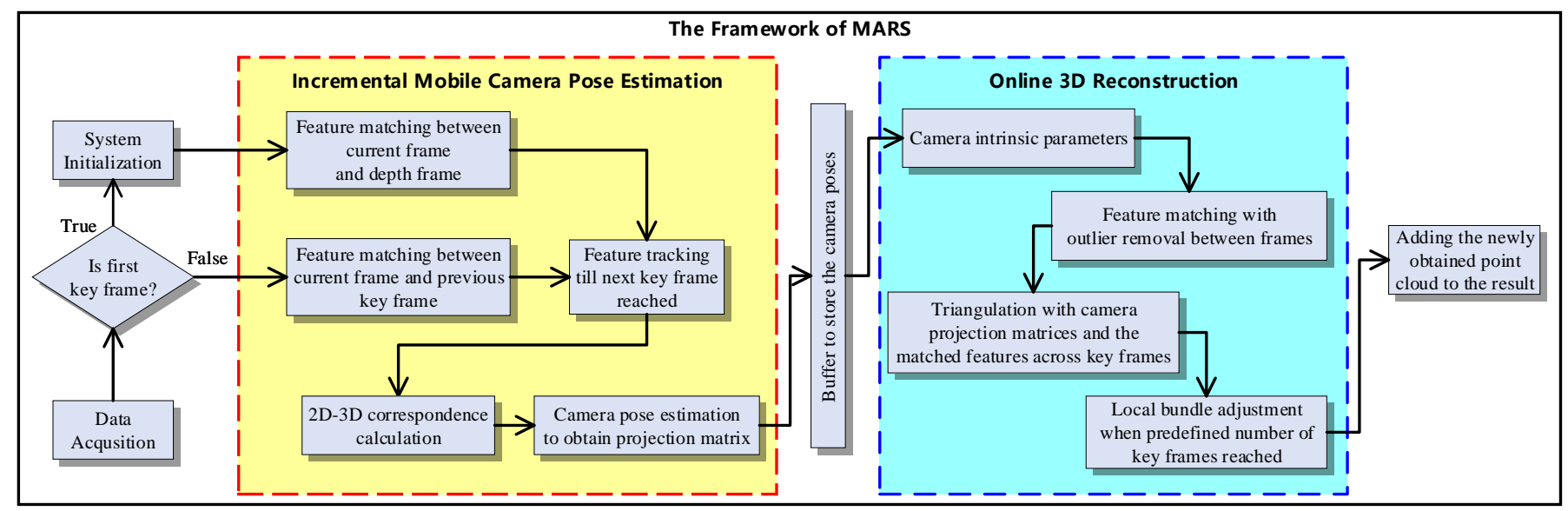

Fig. 1: Demonstration of the data flow of the system proposed in this paper. The yellow and cyan blocks are two parallel processes, in which the arrows indicate the data flows.

based 3D estimation from a single RGB image has also been introduced [12], [13]. However, its accuracy and resolution still need to be improved.

To improve the flexibility without loss in accuracy, this paper presents a solution of Metrically Accurate 3D Reconstruction System (MARS) that combines the advantages from several types of 3D reconstruction methods. The proposed solution utilizes a single mobile RGB camera for 3D perception, which only needs to be initialized once at the beginning of the process with a low-resolution depth map. This depth map can either be estimated from one RGB frame using a machine learning based method or obtained from a stationary depth sensor fixed on the ground. This makes the reconstruction process more flexible. The proposed system can recover the 3D data at the actual scale of the scene, which makes it useful in practical applications. The system can also be functional with a mobile device easily through an onboard mobile RGB camera.

The data flow of the proposed method is shown in Fig. 1 It consists of two parallel processes: Incremental Mobile Camera Pose Estimation, and Metric Online 3D Reconstruction. These two parallel processes are running individually in their own loops after the system being initialized. A buffer that stores the estimated camera poses acts as a bridge to connect those two processes. The arrows in Fig. 1 indicate the data flows running through two processes. The camera pose estimation process is continuously receiving input data from an RGB camera and outputting data to the buffer. The $3 \mathrm{D}$ reconstruction process retrieves data from the buffer in each loop and incrementally adds generated point cloud to the final result. All components in Fig. 1 will be fully discussed in Section 3 and Section 4

The contributions of the proposed method include:

1) A parelleism-based pipeline is presented for online $3 \mathrm{D}$ recovery with a metrical scale. It combines the advantages of both depth sensor based methods and RGB camera based methods.

2) An iterated camera pose estimation process initilalized by a depth map is proposed. It is achieved with weighted 2D-3D correspondence down-sampling based on the re-projection confidence estimation.

3) An iterative outlier removal process is proposed based on weighted randomly down sampling and re-projection for feature matching.

4) A local Bundle Adjustment scheme is adopted for local sequence of Key frames for reconstruction refinement.

The remainder of the paper is organized as follows. Section 2 discusses the related works. Section 3 and Section 4 describe two parallel processes demonstrated in Fig. 1. which are respectively the incremental camera pose estimation, and the metrical 3D reconstruction with an outlier removal scheme for matched features. Experiments are discussed in Section 5 . The paper is concluded in Section 6

\section{Related Work}

Many 3D reconstruction methods have been proposed. According to how the hardware are utilized, these methods can be discussed in following aspects.

\subsection{D perception using dedicated depth hardware}

This type of methods achieves the 3D recovery using a deliberately designed device dedicated for 3D reconstruction tasks. The hardware commonly consists of multiple projectors and sensors. For example, a structured-light based depth sensing system usually consists of a pattern projector and a pattern receiver. They are installed with a noticeable displacement that is large enough for 3D triangulations [14]. Similarly, a laser projector and a receiver are usually included in the laser ranger based depth device with an additional timing module to measure the TOF [15]. Multiple studies on depth hardware or system designs have been conducted recently. Rosen and Kelner [16] investigated into 3D imaging by a self-reference single-channel digital incoherent holography using a hologram recorder. Wei et al. [17] studied methodologies for color 3D imaging with two near-infrared cameras. Besides those studies that directly designed a depth hardware, other researches aiming at enhancing the 3D reconstruction with a consumer-grade depth sensor were also explored. For example, Zhang et al. [18] utilized an RGB-D camera for 3D facial reconstruction using RBF based adaptive interpolation. Albert et al. [19] proposed a RGB-D based system for visual odometry estimation and achieved encouraging results. Their method only focused on ego localization but required a pure rotation 
as an initialization for pose estimation. Angela et al. [20] presented a BundlFusion system for $3 \mathrm{D}$ reconstruction using surface registration. Their reconstruction was obtained by directly moving an RGB-D camera around the scene. Choi et al. [21] presented an offline RGB-D data-based indoor scene reconstruction. Their method took RGB-D video as input and considered geometric registration errors through an optimiztion approach for 3D recovery. Whelan et al. [22] proposed ElasticFusion for dense visual SLAM. They used an RGB-D camera as the input device. A windowed surfelbased fusion along with a non-rigid surface deformation were utilized to achieve scene reconstruction. Newcombe et al. [23] proposed the classic KinectFusion for 3D reconstruction with a moving Kinect. They achieved impressive experiment results. However, due to the size and weight of the Kinect, it's still inconvenient to freely move the Kinect as a handheld sensor in practical applications. In addition, the wires and cables of the Kinect can cause additional restrictions for using the system in many practical applications. If the Kinect was replaced with a depth sensor of a smaller size, the price of the system would be increased accordingly. Though the depth sensors can obtain more accurate 3D data, the complexity of the setups makes the sensor hard to be as compact as a RGB camera without a high cost. Compared to an RGB camera, it is therefore inconvenient and expensive to move a depth sensor around freely in practice.

\subsection{D perception only using a single RGB camera}

This type of methods utilizes a single RGB camera for 3D recovery, such as monocular SLAM and the Multi-View Geometry based SFM. Many researchers have presented their works in this field. For the first time, Davison et al. [10] proposed a single RGB camera based SLAM, namely MonoSLAM. It only utilized a single moving RGB camera to recover the 3D landmark locations and camera ego poses simultaneously. It showed impressive performance. However, an initialization by a predefined square pattern on a planar surface was required for their system to achieve the desired functionality. Moreover, their method only focused on the system ego pose estimations rather than 3D reconstruction. Later on, Newcombe et al. [24] later presented a depth sensing system called DTAM to extend the original MonoSLAM. It only used a single RGB camera as well. Besides a smoothness assumption for the depth, their method also required an initialization based on a circular motion of the sensor aiming at the target. The SFM is another commonly used framework for single RGB camera based methods. Magerand et al. [25] discussed the projective structure from motion and its applications for outdoor 3D reconstruction. Its offline nature could be a potential limitation in certain applications. The Shape from Shading based methods can also retrieve a depth map from the RGB images. However, more constraints and assumptions are required. Since it is an ill-posed problem, the performance is thus unstable [26].

\section{$2.33 \mathrm{D}$ reconstructions based on sensor fusions}

There are also researches that explore the combination of a single RGB camera with another device. For instance, the classic Photometric Stereo based method utilizes a single RGB camera [27]. However, the depth data can only be accurately achieved with multiple directional artificial illuminations. Stereo Vision based systems are commonly used. These systems use two RGB cameras with a noticeable baseline for 3D triangulations [28]. There are some other similar methods, such as the light-field made of multiple RGB cameras [29].

\subsection{Machine learning based 3D perception}

Machine Learning based depth estimation from a single RGB image has been introduced recently and has drawn wide attentions in the $3 \mathrm{D}$ reconstruction community. This type of methods only analyzes a single frame of the RGB data for the depth map estimation. Zhou et al. [12] have presented a unsupervised learning method for depth and ego-motion estimation from visual data. Two convolutional neural networks were employed for depth prediction and pose prediction. The results demonstrated encouraging performance. Yet, the estimated depth was oversmoothed compared to the ground truth, which led to loss of details. He et al. [13] proposed a research for learning depth from single images with deep neural network. Their method was achieved by fusing the middle-level information on the focal length data, which could bring improvement to the performance. However, the final result of their method was still an oversmoothed depth map, which lacked details. Liu et al. [30] proposed a Deep Convolutional Neural Field (DCNF) based depth learning method from a single RGB image. The DCNF model explored the combination of Convolutional Neural Network (CNN) and Continuous Conditional Random Field (CRF) by assuming the depth in an image was continuous. Experiments indicated a better performance than the previous methods. However, the depth map still lacked details. Up to now, although the machine learning based 3D prediction can be visually appealing, it is still less metrically accurate than the methods that are based on the calculation with 3D geometric models.

\section{Incremental Camera pose Estimation}

In this section, the first parallel process in Fig. 1 is discussed, which is the camera pose estimation. The proposed system uses a single freely moving RGB camera for 3D recovery. To calculate the $3 \mathrm{D}$ points in the space, the poses are estimated first for the camera that captures the scene from different view-points. The camera pose determines the camera projection matrix, which describes the relationship between the 3D points in the space and the 2D points projected on an image plane. Perspective-n-Points $(\mathrm{Pn} P)$ based methods are among the most commonly used approaches for this problem. They utilize $n$ known 2D-3D correspondences between the 2D points in the image and 3D points in the space. Most PnPmethods rely on redundant 2D-3D corresponding pairs for a better accuracy. There are two types of definitions for those PnPbased methods: distance-based and transformation-based. They can be expressed as in (1) and (2) respectively:

$$
\begin{gathered}
d_{i 0}^{2}+d_{j 0}^{2}-d_{i 0} d_{j 0} \cos \theta_{i j}=d_{i j}^{2}, \quad i \neq j \\
\mathbf{x}_{i}=\mathbf{K}[\mathbf{R} \mid \mathbf{t}] \mathbf{X}_{i}
\end{gathered}
$$




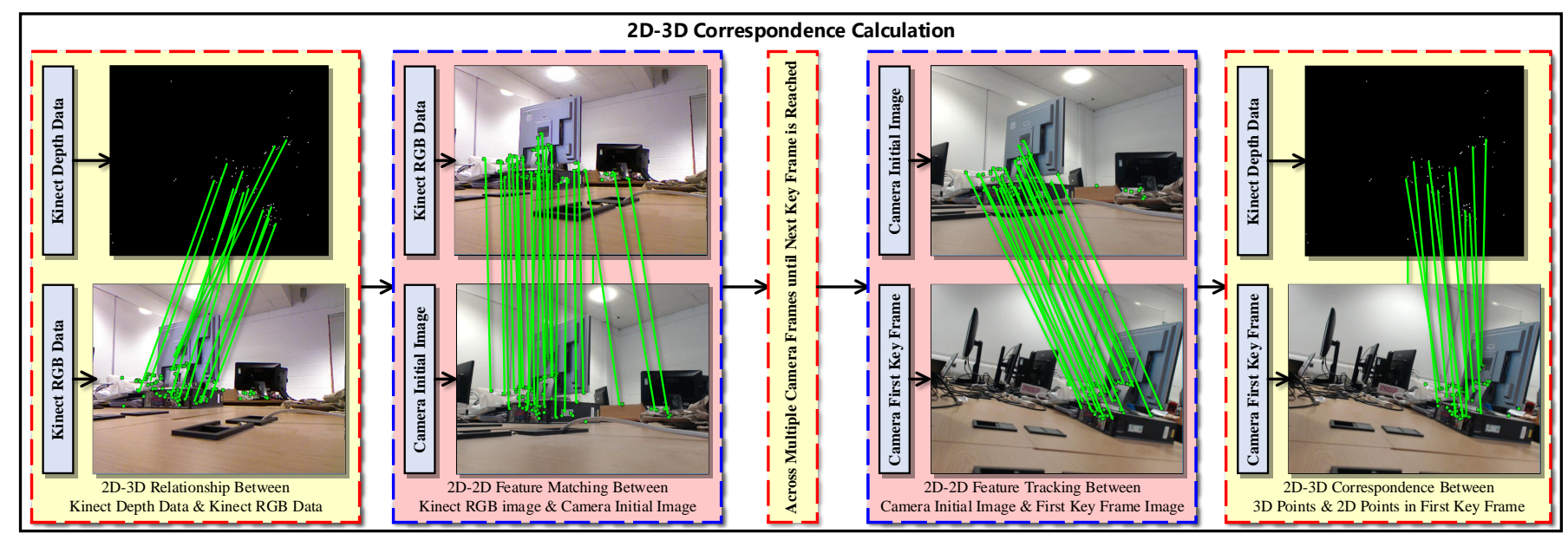

Fig. 2: Illustration for the process of 2D-3D Correspondence Calculation.

In (1), $d_{i j}=\left|\overrightarrow{\mathrm{X}_{i} \mathrm{X}_{j}}\right|$ is the Euclidean distance between two 3D points of $\mathrm{X}_{i}$ and $\mathrm{X}_{j}$. If $\mathrm{O}$ is the camera principal point, then $d_{i 0}$ and $d_{j 0}$ are the lengths of two vectors $\overrightarrow{\mathrm{X}_{i} \mathrm{O}}$ and $\overrightarrow{\mathrm{X}_{j} \mathrm{O}}$ respectively. $\theta_{i j}=\angle \mathrm{X}_{i} \mathrm{OX}_{j}$. In $22, \mathrm{x}_{i}$ is a $2 \mathrm{D}$ projection of the $3 \mathrm{D}$ point $\mathrm{X}_{i}$, while $\mathbf{K}$ is the camera intrinsic parameter matrix. $\mathbf{R}$ and $\mathbf{t}$ are the rotation matrix and translation vector respectively of the camera relative to the coordinate system origin. $\mathbf{x}_{i}, \mathbf{K}$ and $\mathrm{X}_{i}$ are known in prior. Therefore, to estimate the camera poses, the 2D-3D $\left(\mathrm{x}_{i}-\mathrm{X}_{i}\right)$ correspondence should be extracted first.

Concerning the mathematical notations, this paper uses bold capital letter for matrix or image (which is actually a matrix as well), bold small letter for vector, capital letter for 3D point, small letter for 2D point, and italic small letter for scalar value.

\subsection{D-3D correspondence}

The camera pose estimation is an incremental process with two cases: a) to estimate the pose of the RGB camera for the first key frame; b) to estimate the camera poses corresponding to the rest key frames. Since the proposed system is initialized using a depth map, the $3 \mathrm{D}$ points in the aforementioned 2D-3D correspondence for case a) are calculated from the depth map directly. As for case $\mathbf{b}$ ), those $3 \mathrm{D}$ points are from the $3 \mathrm{D}$ reconstructed results obtained using previous key frames.

For the demonstrative purpose in this paper, a Kinect is used to obtain a depth map for the system initialization. Either a RGB-D sensor or a ML based depth estimation process can present a depth map and a RGB image simultaneously. Therefore, the 2D-3D point correspondences for estimating the pose of the first key frame are achieved through an intermediate step, which extracts the 2D-2D point correspondence between the first key frame from the mobile RGB camera and the RGB image from either the RGB-D sensor or the ML based depth estimation algorithm. This process is shown in Fig. 2. As for the rest key frames, since the 3D points contributed to the 2D-3D correspondence are from the $3 \mathrm{D}$ point cloud triangulated using the $2 \mathrm{D}$ points from previous key frames, their corresponding 2D key-points in the rest key frames can be found by the 2D-2D correspondence established according to the feature matching and tracking process. Therefore, the only difference between the case $\mathbf{a})$ and $\mathbf{b}$ ) is that the RGB image from the RGB-D sensor for the situation a) is replaced by the previous key frame for the situation a). The Kinect's RGB image is treated as the initial $\left(0^{t h}\right)$ key frame for algorithmic convenience.

The 2D-2D correspondence between the current and the next key frame is calculated in two stages: a) the feature matching between the current key frame and the first nonkey frame that follows; and a) the feature tracking on the non-key frames while the RGB camera is moving towards the next key frame.

The feature matching process is conducted between the key frame and its first following consecutive non-key frame. This process generates potential 2D-2D correspondence pairs before the camera reaches the position of the next key frame. The feature matching is utilized in this stage since the viewpoint of the first following non-key frame is spatially similar to the current key frame. This makes the matching results more reliable. Directly conducting the feature matching between two key frames can cause more mis-matchings and error-matchings due to the potential large viewpoint difference. The errors can also be further accumulated in the following feature tracking process. The mis-matching and error-matching will be further discussed in Section 4.1. where an outlier removal process is proposed to refine the feature matching results.

The proposed MARS method achieves feature detection using Pyramid FAST as extractor and the Rotated BRIEF as descriptor. This scheme is also used in ORB [31]. The feature description can be treated as a binary string. The matching is done by greedily searching among all possible binary tests

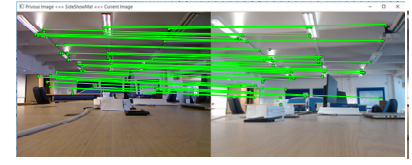

(a)

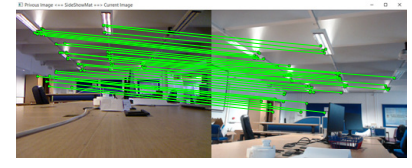

(b)
Fig. 3: Illustration of feature matching and tracking between Kinect RGB image (left) and camera frame (right). (a) Matching with Outlier Removal when camera is in initial position; (b) Tracking while camera moves. 


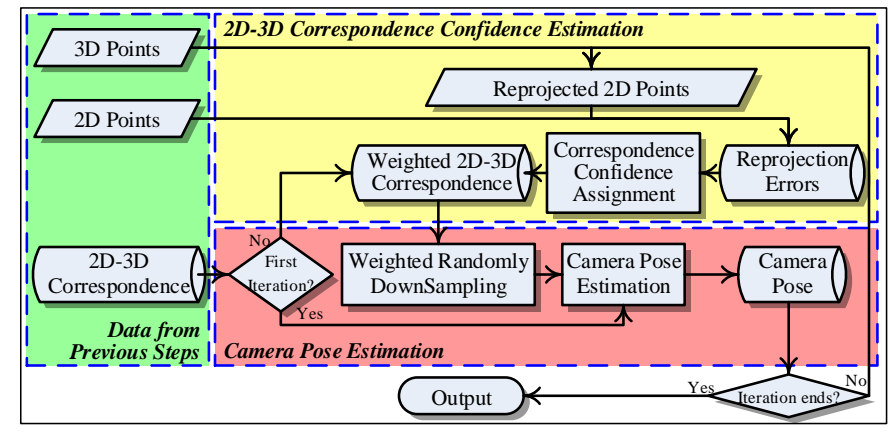

Fig. 4: The workflow of the iterated camera pose estimation with random down-sampling of the 2D-3D correspondence. of the candidate descriptors in Hamming distance.

Following the feature matching results, the feature tracking is applied to maintain the established 2D-2D correspondence while the camera is moving towards the next key frame. The optical flow is utilized in this process. In Fig. 3. an example to demonstrate a maintenance of feature correspondence with a moving camera is illustrated. Once the 2D-2D point correspondence between two key frames is found, the $2 \mathrm{D}-3 \mathrm{D}$ point correspondence can be achieved easily.

\subsection{Iterated camera pose estimation}

The camera pose consists of two components: The Rotation (R) and the Translation (t) of the camera principal point (camera coordinate system) with respect to the world coordinate system in 3D space. Based on the camera projection model, the projection matrix can be obtained by combining both $\mathbf{R}$ and $\mathbf{t}$. As shown in (3), $\mathrm{P}$ denotes the projection matrix, while $(u, v, 1)^{\mathrm{T}}$ and $(x, y, z, 1)^{\mathrm{T}}$ respectively refer to the $2 \mathrm{D}$ point coordinate on the camera image (camera coordinate system) and the 3D point coordinate in the space (world coordinate system). $\mathbf{K}$ is a camera intrinsic matrix that contains the intrinsic parameters of the camera.

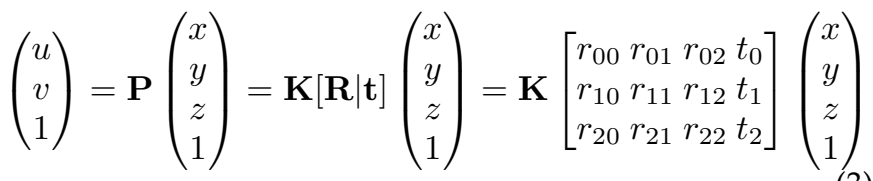

Therefore, according to (3), with the knowledge of the 2D-3D point correspondence, an optimization process can be formulated to estimate the camera pose, as 4 demonstrated.

$$
\underset{\{\mathbf{R}, \mathbf{t}\}}{\arg \min } \sum_{i=1}^{N_{2 d 3 d}}\left\|\mathbf{K}[\mathbf{R} \mid \mathbf{t}] \mathbf{X}_{i}-\mathbf{x}_{i}\right\|
$$

where the point pair of $\mathrm{x}_{i}$ and $\mathrm{X}_{i}$ is the ith corresponding $2 \mathrm{D}-3 \mathrm{D}$ point pair in the whole $2 \mathrm{D}-3 \mathrm{D}$ point correspondence dataset. Inspired by the EPnP |32|, the number of the pairs in the 2D-3D point correspondence dataset is virtually increased to maximize the stability of the estimation. It is achieved by taking into account the 2D-3D centroid pairs that are calculated from each four 2D-3D corresponding pairs. These 2D-3D centroid pairs are also added to the 2D3D point correspondence.

The intrinsic parameters of the camera can be obtained with the camera calibration process. This can be achieved by

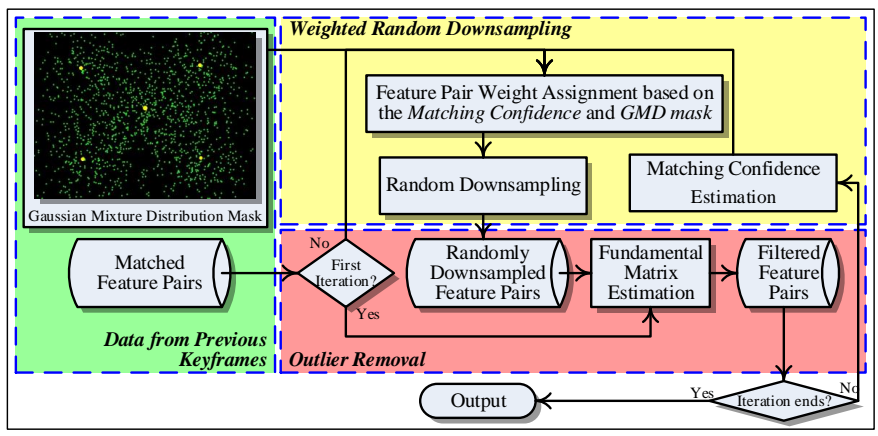

Fig. 5: The workflow of the outlier removal process. The topleft corner is the GMD-RDS mask, where the yellow dots are the means of the GMD.

either capturing a checkerboard from multiple viewpoints, or the EXIF data extracted from the image frames. A camera with a fixed focal length is utilized in the proposed system to ensure a consistent $\mathbf{K}$ while the camera is moving.

In the proposed system, the camera pose estimation is achieved through an iterative process. As shown in Fig. 4 to minimize the estimation errors, in the first iteration, the camera pose is estimated by all 2D-3D correspondence pairs obtained in previous step. It assumes that the majority of those 2D-3D pairs is inliers. The 3D point in each pair is then projected as a $2 \mathrm{D}$ point on the image plane using the estimated camera pose in the current loop. By comparing the projected $2 \mathrm{D}$ points with the original 2D points from the 2D-3D correspondence pairs, a reprojection error for each point can be estimated. According to this reprojection error, a weight is assigned to each 2D-3D pair as a confidence for this correspondence pair. The outliers are assigned accordingly in the current iteration with a threshold based on the correspondence confidences. If the number of the outliers is greater than a threshold, the 2D-3D pairs will be randomly down-sampled (RDS) according to their assigned confidences. Thus, the pairs with lower reprojection errors are more likely to be sampled. This down-sampled correspondence set is used for the next iteration. The iteration stops once either of these two criteria is reached: a) the number of the outliers is below a threshold, or $\mathbf{b}$ ) the max iteration number is reached.

\section{Online 3D Reconstruction}

The second parallel process in Fig. 1 aims to reconstructing the $3 \mathrm{D}$ points with the $2 \mathrm{D}$ features tracked across camera key frames. However, for most cases, those matched features are full of outliers due to the image noise, camera distortion, and many other reasons. Hence, the proposed method includes a confidence based outlier removal scheme combined with a GMD-RDS strategy, which can reduce the impacts of the outliers to the accuracy of the following triangulation process.

\subsection{Outlier removal for feature matching}

The feature matching process for the $3 \mathrm{D}$ point triangulations is conducted regardless of the matching results in the camera pose estimation step. The matched 2D point pairs in this step are contributed to the calculation of new 3D points, rather than the known 3D points for camera pose 
estimation. Moreover, the view-points between key frames in this step are larger, which can lead to more mis-matchings and error-matchings. This will affect the accuracy of the triangulation afterward. To tackle this problem, an Outlier Removal process is proposed to filter the feature matching results. This is an iterative process using a filter based on the Fundamental Matrix $(\mathbf{F})$.

The $\mathbf{F}$ Based filtering is described in $(6)$, where $\mathbf{H}$ is the transformation between two 2D points of $x$ and $x^{\prime}$. Parameter $e$ and $e^{\prime}$ are the 3D points of epipoles. $l^{\prime}$ is the epipolar line. [ $]_{\times}$is the cross products operator.

$$
\left\{\begin{array}{l}
\mathbf{x}^{\prime}=\mathbf{H} \mathbf{x} \\
l^{\prime}=\left[e^{\prime}\right]_{\times} \mathbf{x}^{\prime}=\left[e^{\prime}\right]_{\times} \mathbf{H} \mathbf{x}=\mathbf{F} \mathbf{x} \\
\mathbf{F}=\left[e^{\prime}\right]_{\times} \mathbf{H}
\end{array}\right.
$$

$\mathbf{F}$ is a $3 \times 3$ matrix describing the projective relationship between two images. As shown in Fig. 6a. each pair of the accurately matched 2D points should be two projections from a same 3D point. $F$ implies that these 2D points should be on a same epipolar plane. The intersections of this epipolar plane and the image is called the epipolar line $\left(l^{\prime}\right)$. Since these two matched 2D points should comply with (6), $\mathbf{F}$ can be used as a filter to refine the matching results by checking the distance of the point from its epipolar line defined by its correspondence.

$$
\mathrm{x}^{\prime \mathrm{T}} \mathbf{F x}=0
$$

It is determined that the matched pairs should follow the model described by 6 . Thus, rather than simply applying the RANSAC based solutions, the proposed outlier removal step utilizes $\mathbf{F}$ as the filter model to avoid the uncertainty during the model estimating process in RANSAC based methods.

The estimation of $\mathbf{F}$ can be done easily. However, the performance of this filter is still limited since the estimate of $\mathbf{F}$ is also based on the same $2 \mathrm{D}$ point pairs that need refinements. Thus, MARS employs an iterative filtering process as shown in Fig. 5. At the end of each iteration, a confidence is assigned to each point pairs according to the epipolor constraint described by the estimated $\mathbf{F}$, as show in (7). The higher the confidence of a pair, the more likely it is to be sampled for the next iteration.

$$
\text { confidence }_{i}=1-\frac{\mathrm{x}_{i}^{\prime T} \mathbf{F} \mathbf{x}_{i}}{\sum_{j}^{\text {All matched pairs }} \mathrm{x}_{j}^{\prime T} \mathbf{F} \mathbf{x}_{j}}
$$

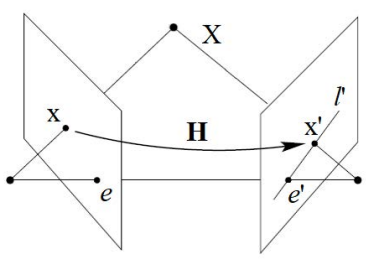

(a)

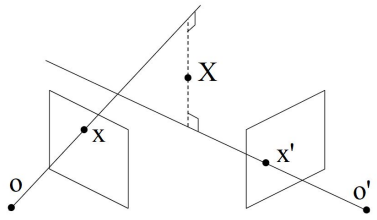

(b)
Fig. 6: (a) Illustration for the geometric relationship of matched 2D point pair; (b) The principle of the triangulation process with the case of two frames.
Additionally, we have found that the outliers are more likely to occur near the image borders due to the camera lens distortion, and the occlusions by the image edges. Thus, a Random Down-Sampling (RDS) process is applied to collect more matched pairs around the image center and less near the image borders. Gaussian Mixture Distribution (GMD) is used in this process. The confidence of a matched pair is considered.

As shown in (8), $G\left(x, y, \sigma_{i}\right)$ refers to a Gaussian distribution for sampling the point at position $(x, y)$ of an image. With the RDS process, the coordinates of the sampled feature pairs in one image follow the distribution of $G\left(x, y, \sigma_{i}\right)$.

$$
G\left(x, y, \sigma_{i}\right)=\frac{1}{2 \pi \sigma_{i}^{2}} e^{\frac{-\left(\left(x-x_{\text {mean }}\right)^{2}-\left(y-y_{\text {mean }}\right)^{2}\right)}{2 \sigma_{i}^{2}}}
$$

As shown in top-left corner of Fig. 5, the mean of the main Gaussian distribution is seated at the image center with a standard deviation of 70. To sample as many matched pairs as possible, another four Gaussian distributions with their means seated around the four corners of the image are included with each standard deviation being 80 .

\subsection{Triangulation with ICP corrections}

With the projection matrices and matched pairs obtained across multiple key frames, the triangulation process is conducted for 3D point calculations. Fig. $6 \mathrm{~b}$ demonstrates a two-frame case of the triangulation process, where $x$ and $\mathrm{x}^{\prime}$ are two $2 \mathrm{D}$ points projected onto two camera images from a same $3 \mathrm{D}$ point $X$ in the space. The optical centers of these two cameras are indicated by o and $\mathrm{o}^{\prime}$ respectively. Ideally, the two beams of ox and $o^{\prime} x^{\prime}$ should intersect at the position of $X$. However, due to projection errors and other deviations, these two beams can never intersect. The aim of triangulation is to find the closest point between these two beams. It can be solved by a linear optimization process, which is described in 9 .

$$
\left\{\begin{array}{l}
\mathbf{x}=\mathbf{P} \mathbf{X} \\
w u=\mathbf{P}_{1}^{\mathrm{T}} \mathbf{X}, \quad w v=\mathbf{P}_{2}^{\mathrm{T}} \mathbf{X}, \quad w=\mathbf{P}_{3}^{\mathrm{T}} \mathbf{X} \\
u \mathbf{P}_{3}^{\mathrm{T}} \mathbf{X}=\mathbf{P}_{1}^{\mathrm{T}} \mathbf{X}, \quad v \mathbf{P}_{3}^{\mathrm{T}} \mathbf{X}=\mathbf{P}_{2}^{\mathrm{T}} \mathbf{X} \\
{\left[(u+v) \mathbf{P}_{3}^{\mathrm{T}}-\mathbf{P}_{1}^{\mathrm{T}}-\mathbf{P}_{2}^{\mathrm{T}}\right] \mathbf{X}=0}
\end{array}\right.
$$

where $\mathbf{P}$ is a $3 \times 4$ projection matrix. Each $\mathbf{P}_{i}^{\mathrm{T}}$ is the $i^{\text {th }}$ row of $\mathbf{P} . w$ is a scale factor for the coordinates. With the second line in (9), this $w$ can be elimated. By combining the two terms in the third line, $\left[(u+v) \mathbf{P}_{3}^{\mathrm{T}}-\mathbf{P}_{1}^{\mathrm{T}}-\mathbf{P}_{2}^{\mathrm{T}}\right]$ can be derived, which is obviously a $1 \times 4$ row vector. In practice, one $3 \mathrm{D}$ point can be projected onto multiple 2D planes of different key frames during the reconstruction process. It is commonly called a projection track of a $3 \mathrm{D}$ point across multiple frames. This can be achieved by concatenating matched 2D features through these key frames. Thus, with this projection track of the same 3D point across multiple key frames, a linear system $\mathbf{A X}=\mathbf{0}$ can be formed up by the fourth row of 9 ). In addition, since the 3D point $X$ is represented using a homogeneous coordinate, the linear system $A X=0$ can be easily converted into the form of $\mathbf{A}^{\prime} \mathbf{X}^{\prime}=\mathbf{B}$ by a fixed scale factor in this homogeneous coordinate, as described in (10). By solving this linear system 


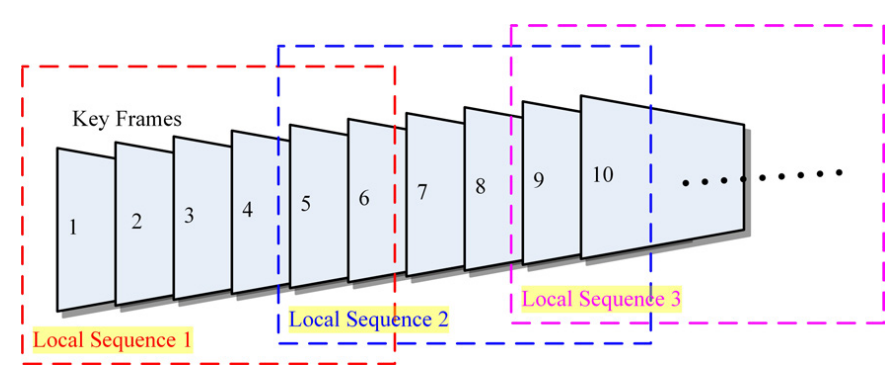

Fig. 7: Illustration of local sequences for consecutive key frames.

with $n$ projected 2D points from the same 3D point shown in (10), the triangulation can be achieved.

$$
\begin{aligned}
& \left\{\mathbf{A X}=\left[\begin{array}{llll}
\mathbf{a}_{0} & \mathbf{a}_{1} & \mathbf{a}_{2} & \mathbf{a}_{3}
\end{array}\right]\left[\begin{array}{llll}
x & y & z & d
\end{array}\right]^{\mathrm{T}}=\mathbf{0}\right. \\
& \left\{\mathbf{a}_{i}=\left[\begin{array}{llll}
a_{0 i} & a_{1 i} & a_{2 i} & a_{3 i} \ldots a_{n i}
\end{array}\right]^{\mathrm{T}}\right. \\
& \Rightarrow\left[\begin{array}{lll}
\mathbf{a}_{0} & \mathbf{a}_{1} & \mathbf{a}_{2}
\end{array}\right]\left[\begin{array}{lll}
x & y & z
\end{array}\right]^{\mathrm{T}}=-d \mathbf{a}_{3}^{\mathrm{T}}
\end{aligned}
$$

Once the 3D points are triangulated with projection matrices, an Iterated Closest Points (ICP) process is applied to correct the slight shifts between the triangulated 3D points and the previous known 3D point cloud. The 3D point positions and the current camera pose are refined simultaneously. Since the 3D points used for initialization are based on the depth map, there is no scale ambiguity. A fixed scale factor can be used in (10). The 3D points obtained from the rest key frames are also metrical scaled accordingly.

\subsection{Local bundle adjustment}

Since the process of the camera pose estimation and the 3D reconstruction is incremental, it will inevitably cause error accumulation during the process, which can lead to a drift in the results. In the MARS, since the loop closure cannot be guaranteed in close-range scenarios, following the ICP refinement, a Local Bundle Adjustment (LBA) scheme is proposed to locally and incrementally optimize the result. This helps to reduce error accumulation. The LBA is applied on the local sequences composed of multiple key frames. In our system, a local sequence consists of 20 consecutive key frames. 5 or more consecutive frames are allowed to be overlapped with neighboring local sequences, as illustrated in Fig. 7

A series of optimization processes are applied for the sequences of key frames. As shown in (11), the estimated camera projection matrices and the $3 \mathrm{D}$ point coordinates are treated as an initialization for the optimization process.

$$
\underset{\{\mathbf{P}, \mathrm{X}\}}{\arg \min } \sum_{i \in \text { keyframe index }} \sum_{j \in 3 \mathrm{D} \text { point index }}\left\|\mathbf{P}_{i} \mathrm{X}_{j}-\mathrm{x}_{i} j\right\|
$$

where $\mathbf{P}_{i}$ is a projection matrix for $i^{\text {th }}$ key frame. $\mathbf{X}_{j}$ is $j^{t h}$ 3D point that is visible in $i^{t h}$ key frame. $x_{i} j$ is a $2 \mathrm{D}$ projection of $j^{t h}$ 3D point on the $i^{t h}$ key frame. It jointly refines the camera poses $\left\{\mathbf{P}_{i}\right\}$ and the $3 \mathrm{D}$ point cloud $\left\{\mathrm{X}_{j}\right\}$.

\section{EXPERIMENTS}

The proposed method is tested on both real-world data and public datasets with comparisons with several existing methods. The experiments are conducted on a laptop with implementation in $\mathrm{C}++$.

\subsection{Experiments with real-world data}

In this part of the experiments, the experiment data for the proposed system are obtained from a Kinect and a handy webcam in real time. Samples of the experiments are demonstrated in Fig. 8 . In the experiments, the MARS is initialized using a depth map from the Kinect (the $2^{\text {nd }}$ row or $4^{\text {th }}$ row in Fig. 8). Only the depth data within a range of 1.5 meters from the sensor is utilized for the initialization. This is because the depth data with a larger distance are less metrical stable. It thus, contributes less to the camera pose estimation during the initialization. The key frame lengths are only 23 and 39 for two experiments respectively to reconstruct target objects along with a part of the background around them, as shown in Fig. 8 . The $5^{\text {th }}$ row and $3^{r d}$ row in Fig. 8 containing the first few frames in the key frame sequences illustrate the two scenarios of the 2D3D point correspondence establishment and the two-stage feature tracking described in Section 3.1. The outlier removal procedures for both 2D-3D correspondence and 2D feature matching can effectively enhance the tracking performance.

The first image in either the $2^{\text {nd }}$ or the $4^{t h}$ row of Fig. 8 is a depth map of the scene from the RGB-D sensor. It is used for initialization. Due to occlusions and limited FOV, the depth map is incomplete. For example, some parts of the carpet behind the box is invisible because of the occlusion. However, after initialization from such a depth map, the reconstructed 3D point clouds can easily cover those blank areas with only 23 and 39 key frames respectively, as shown by the rest of the images in the $2^{n d}$ and $4^{\text {th }}$ row of Fig. 8 .

Apart from the visual demonstrations, the performance of the proposed method is also assessed by evaluating the metric stability. The calculation of this evaluation is achieved using a quotient of two quantities of a same length in the reconstructed point cloud in two different units, as shown in (12).

$$
r_{\text {pixel }_{m} \text { etrix }}=\text { lengt }_{\text {pixel }} / \text { lengt } h_{\text {metric }}
$$

where length $h_{\text {metric }}$ is a metric distance measurement in millimetres, while length $h_{\text {pixel }}$ is the value of the same distance in pixel units measured using coordinate values. The $r_{\text {pixel }}$ etrix is evaluated for several different positions in the reconstructed point cloud. The stability of $r$ in different locations reflects the performance of the metric reconstruction. For example, the 3D reconstructions by the classic SFM have different scale factors relative to the real world when they are obtained in different scenarios. This means the SFM has an unstable rpixel/metric.

As shown in Fig. 9, the proposed MARS is compared against Kinect in terms of the metrics stability based on the evaluations of rpixel/metric. Due to the mechanism of the structure light sensor, a rpixel/metric is expected for the Kinect as about 21.606 pixel per $\mathrm{mm}$ in average. It is consistent with the experiment results shown in Fig. 9 As for MARS, since it is initialized by Kinect's depth map, the reconstructions follow a similar but steadier evaluation of $r_{\text {pixel }}$ etrix .

The $r_{\text {pixel }}$ etrix evaluation varies for the Kinect according to how far the reconstructed 3D points are from the sensor. As shown in 13$)$, if $\left(u_{0}, v_{0}\right)$ is the principal point of the depth sensor, $f$ is the focal length, and $z$ is the depth 

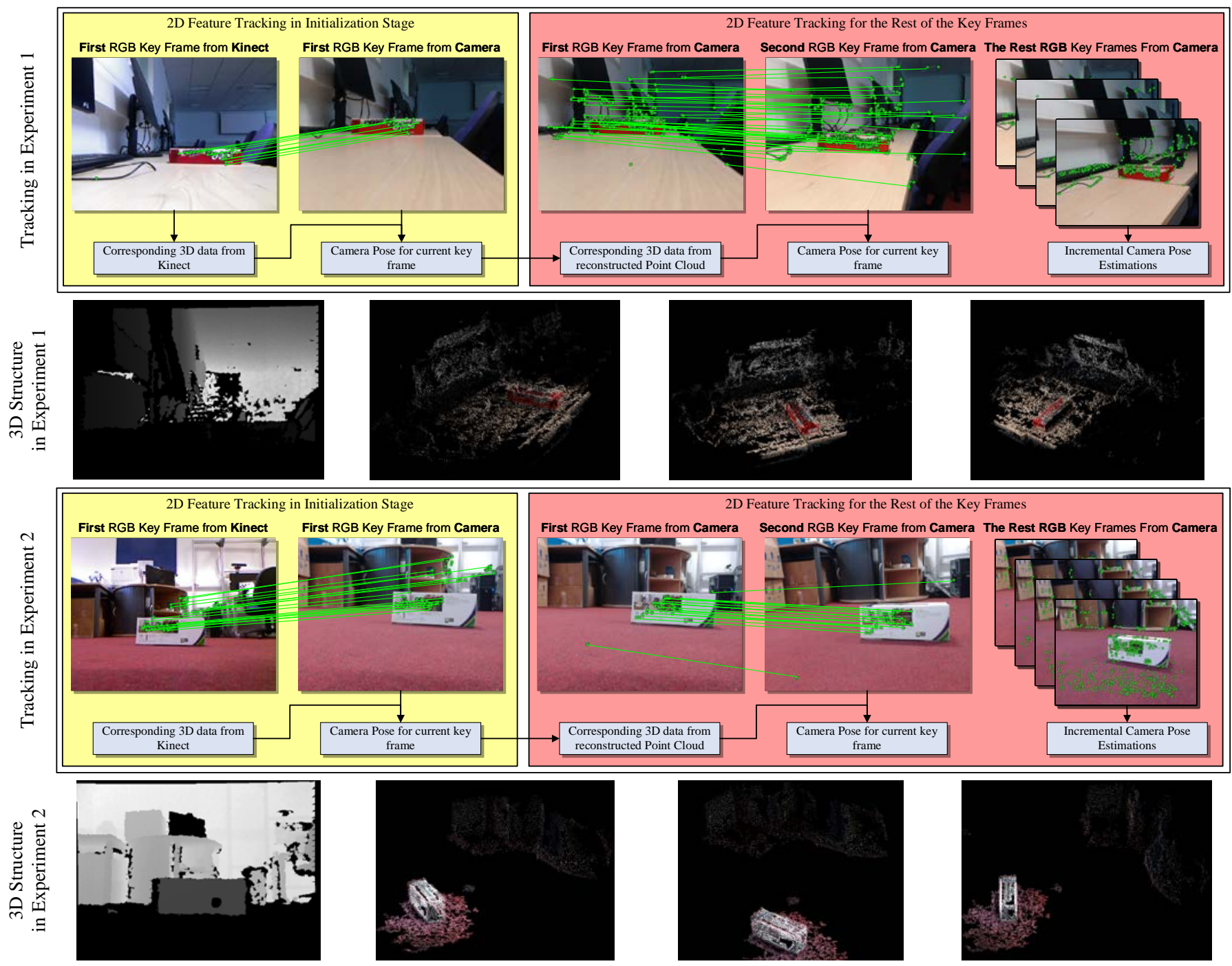

Fig. 8: Illustration of two experiment examples for the 3D reconstruction of the paper. The 1st row and the 3rd row are the first few key frames with tracked 2D points for camera pose estimations. They illustrate the two stages of the tracking process. The first image in either the 2nd or the 4th row is the depth map as an initialization for the system. The rest three images in either the 2nd or the 4th row show the 3D point cloud of the object reconstructed by the proposed method.

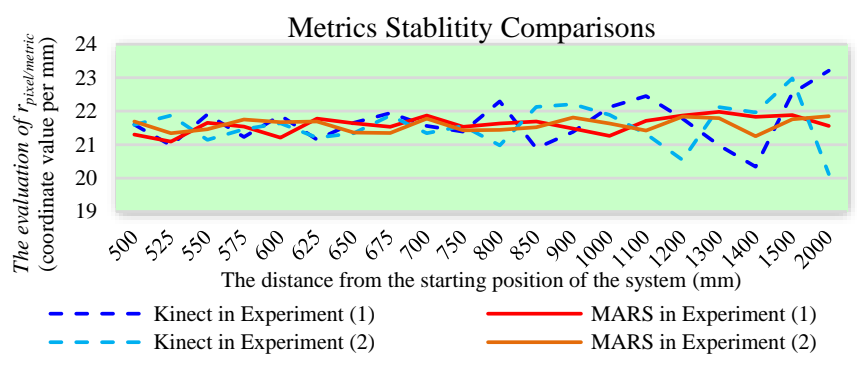

Fig. 9: The comparisons of the system stability. The results are obtained by manually measuring the object in real world and calculating the corresponding distance of the 3D coordinates in point cloud from experiments shown in Fig. 8

value at the location of $(u, v)$ in depth map, then $(x, y, z)$ is a $3 \mathrm{D}$ point corresponding to a $2 \mathrm{D}$ point $(u, v)$ in depth map. Obviously, as $z$ increases, the deviations of $(x, y, z)$ estimates are more likely to be magnified. It thus leads to a greater instability of rpixel/metric for the $3 \mathrm{D}$ point that is
TABLE 1: COMPARISONS OF THE RECONSTRUCTION ACCURACY WITH THE SYNTHETIC ICL-NUM DATASET

\begin{tabular}{ccccc}
\hline \hline & \multicolumn{3}{c}{ Mean distance of the reconstruction to the ground } \\
truth for different datasets
\end{tabular}

far away from the RGB-D sensor.

$$
\frac{x}{u-u_{0}}=\frac{y}{v-v_{0}}=\frac{z}{f}
$$

Since the proposed system is initialized by the $3 \mathrm{D}$ points calculated from a RGB-D sensor in a short range, the $r_{\text {pixel }_{m} \text { etrix }}$ is steadier than Kinect shown in Fig. 9. This 
TABLE 2: THE PROPERTIES OF THE SYNTHETIC ICL-NUM DATASET

\begin{tabular}{cccc}
\hline \hline datasets & Frames & $\begin{array}{c}\text { Trajectory } \\
\text { length }\end{array}$ & $\begin{array}{c}\text { Approximate Number of } \\
\text { reconstructed points using MARS }\end{array}$ \\
\cline { 2 - 4 } lr kt0 & 1509 & $6.54 \mathrm{~m}$ & 144367 \\
lr kt1 & 966 & $2.05 \mathrm{~m}$ & 93534 \\
lr kt2 & 881 & $8.43 \mathrm{~m}$ & 86912 \\
lr kt3 & 1241 & $11.32 \mathrm{~m}$ & 135762 \\
\hline \hline
\end{tabular}

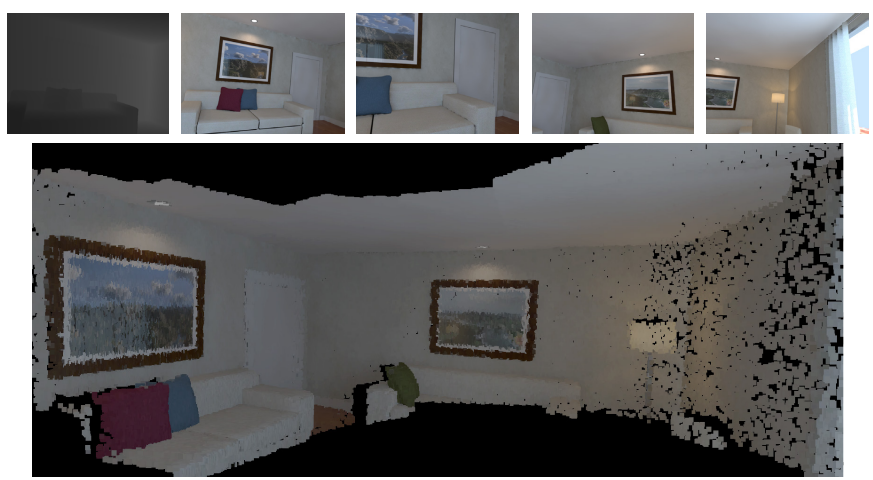

Fig. 10: An illustration of the reconstruction using MARS on the synthetic ICL-NUM datasets. Some of the RGB and depth data from the datasets are demonstrated in the first row.

stable performance holds even for the points far from the system initial position.

\subsection{Performance comparisons on public datasets}

The performance of the proposed MARS is also compared against other state-of-the-art methods including the online systems (DVO-Slam [33], RGB-D Slam [34], MRSMap [35], Kintinuous [36], ElasticFusion [22], BundleFusion [20]) and the offline system (Redwood [21]), as shown in Table1] The comparisons are conducted on the public datasets of the synthetic ICL-NUM provided by Handa et al. [37]. There are 1509, 966, 881 and 1241 frames respectively for those four datasets shown Table2. All those methods utilize RGB$\mathrm{D}$ data to achieve the $3 \mathrm{D}$ reconstructions. Among them, Redwood only uses the depth data, which means the texture of the scene is lost. The rest methods use both the depth and RGB data, which means those methods require a moving RGB-D sensor with high mobility for the reconstruction process in practice.

In the meanwhile, the proposed system only utilizes the RGB data with just one single depth map from the first frame to achieve the reconstruction. This makes the proposed system more flexible in practice. The only moving sensor is a RGB camera. It is still pricy to integrate moving depth sensor into a portable system in practice. Moreover, by only using RGB data after the first frame, the proposed system can still produce competitive reconstruction results compared against other existing methods. It outperforms the majority of other methods even though it ignores the depth data after the first frame. However, it is still very trick for RGB data-based camera pose estimation when large rotations and tiny translations exist in the data sequences. The proposed method can also well handle this problem. The reconstruction using the proposed method is visually demonstrated in Fig. 10

\section{Conclusion}

In this paper, a Metrical Accurate 3D Reconstruction System using a mobile monocular RGB camera is presented. The proposed solution only utilizes a simple initialization by a single depth map for metrical scale calculation. Only a single RGB camera is employed freely moving in the space for $3 \mathrm{D}$ scene sensing. Although there is a range of dedicated depth sensor that can be used for 3D sensing, such as the ones using laser ranger or structured light. They can still be expensive for compactness in practice. Even for those multi-lenses equipped smart phones available in the market, their price can easily reach up to hundreds of Pounds today. Additionally, dedicated depth sensor systems commonly take much more space with extra system complexities than a single RGB camera system. For example, some of them may require a noticeable displacement between system components for its proper functionality when using laser projector and receiver. On the other hand, a single high-resolution camera can always be much cheaper than those depth sensors. The system cost of the proposed method can be as cheap as less than a hundred Pounds in today's market. It can be integrated into other systems without increasing too much system price, such as micro-drones. Compared to those dedicated depth sensor system-based strategies, the proposed solution has a different target group of benefit recipients, which requires lightweight, low cost, and less system complexity in its applications.

The proposed system has been effectively tested on a mid-range laptop. It can achieve competitive reconstruction performance compared with depth data enhanced methods. By only utilizing an RGB camera, the proposed method successfully improves the system flexibility while maintaining a high performance in accuracy. The average mean error distance is less than $10 \mathrm{~mm}$. Once initialized, the feature matching and tracking are activated to establish 2D-3D point correspondence across frames. It is used for incremental camera pose estimation on an iterative process. The feature matching results are refined by a confidence-based Outlier Removal process. The 3D point triangulations are conducted along with a Local Bundle Adjustment strategy to extract a further optimized result. A parallelism-based solution is adopted to organize the two components of camera pose estiamtion and $3 \mathrm{D}$ reconstruction. A buffer stores estimated camera pose acts as an bridge to provide data exchange bewteen those two parallel process. Experiments on both real-world data and public datasets have shown competitive results and performance compared with several state-of-the-art methods.

\section{References}

[1] S. Meerits, V. Nozick, and H. Saito, "Real-time scene reconstruction and triangle mesh generation using multiple RGB-D cameras," Journal of Real-Time Image Processing, 2017.

[2] V. Angladon, S. Gasparini, V. Charvillat, T. Pribanic, T. Petkovic, M. Donlic, B. Ahsan, and F. Bruel, "An evaluation of real-time RGB-D visual odometry algorithms on mobile devices," Journal of Real-Time Image Processing, vol. 16, no. 5, pp. 1643-1660, 2019.

[3] Z. Lv, T. Yin, X. Zhang, H. Song, and G. Chen, "Virtual Reality Smart City Based on WebVRGIS," IEEE Internet of Things Journal, vol. 3, no. 6, pp. 1015-1024, 2016.

[4] Y. Sun, H. Song, A. J. Jara, and R. Bie, "Internet of Things and Big Data Analytics for Smart and Connected Communities," IEEE Access, vol. 4, pp. 766-773, 2016. 
[5] Y. Dong, L. Song, R. Xie, and W. Zhang, "Real-time UHD video super-resolution and transcoding on heterogeneous hardware," Journal of Real-Time Image Processing, 2019.

[6] H. Song and M. Brandt-Pearce, "Range of influence and impact of physical impairments in long-haul DWDM systems," Journal of Lightwave Technology, vol. 31, no. 6, pp. 846-854, 2013.

[7] -, "A 2-D Discrete-Time Model of Physical Impairments in Wavelength-Division Multiplexing Systems," Journal of Lightwave Technology, vol. 30, no. 5, pp. 713-726, 2012.

[8] D. Jiang, W. Wang, L. Shi, and H. Song, "A Compressive SensingBased Approach to End-to-End Network Traffic Reconstruction," IEEE Transactions on Network Science and Engineering, p. 1, 2018.

[9] H. Song, "Digital image watermarking method based on DCT and fractal encoding," IET Image Processing, vol. 11, no. 10, pp. 815821(6), 2017.

[10] A. J. Davison, I. D. Reid, N. D. Molton, and O. Stasse, "MonoSLAM: Real-time single camera SLAM," Pattern Analysis and Machine Intelligence, IEEE Transactions on, vol. 29, no. 6, pp. 1052-1067, 2007.

[11] G. Klein and D. Murray, "Parallel tracking and mapping for small AR workspaces," in Mixed and Augmented Reality, 2007. ISMAR 2007. 6th IEEE and ACM International Symposium on. IEEE, 2007, pp. 225-234.

[12] T. Zhou, M. Brown, N. Snavely, and D. G. Lowe, "Unsupervised Learning of Depth and Ego-Motion From Video," in The IEEE Conference on Computer Vision and Pattern Recognition (CVPR), jul 2017.

[13] L. He, G. Wang, and Z. Hu, "Learning Depth From Single Images With Deep Neural Network Embedding Focal Length," IEEE Transactions on Image Processing, vol. 27, no. 9, pp. 4676-4689, 2018.

[14] M. Rodríguez-Martín, P. Rodríguez-Gonzálvez, D. GonzálezAguilera, and J. Fernández-Hernández, "Feasibility Study of a Structured Light System Applied to Welding Inspection Based on Articulated Coordinate Measure Machine Data," IEEE Sensors Journal, vol. 17, no. 13, pp. 4217-4224, jul 2017.

[15] A. Adam, C. Dann, O. Yair, S. Mazor, and S. Nowozin, "Bayesian Time-of-Flight for Realtime Shape, Illumination and Albedo," IEEE Transactions on Pattern Analysis and Machine Intelligence, vol. 39, no. 5, pp. 851-864, may 2017.

[16] J. Rosen and R. Kelner, "Three-dimensional imaging by selfreference single-channel digital incoherent holography," IEEE Transactions on Industrial Informatics, vol. 12, no. 4, pp. 1571-1583, 2016.

[17] S. Wei, C. Zhou, S. Wang, K. Liu, X. Fan, and J. Ma, "Colorful 3-D Imaging Using an Infrared Dammann Grating," IEEE Transactions on Industrial Informatics, vol. 12, no. 4, pp. 1641-1648, 2016.

[18] S. Zhang, H. Yu, T. Wang, L. Qi, J. Dong, and H. Liu, "Dense 3D facial reconstruction from a single depth image in unconstrained environment," Virtual Reality, vol. 22, no. 1, pp. 37-46, mar 2018.

[19] A. S. Huang, A. Bachrach, P. Henry, M. Krainin, D. Maturana, D. Fox, and N. Roy, "Visual odometry and mapping for autonomous flight using an RGB-D camera," in Robotics Research. Springer, 2017, pp. 235-252

[20] A. Dai, M. Nie \ssner, M. Zollhöfer, S. Izadi, and C. Theobalt, "BundleFusion: Real-Time Globally Consistent 3D Reconstruction Using On-the-Fly Surface Reintegration," ACM Trans. Graph., vol. 36, no. 3, pp. 24:1-24:18, may 2017.

[21] S. Choi, Q.-Y. Zhou, and V. Koltun, "Robust Reconstruction of Indoor Scenes," in The IEEE Conference on Computer Vision and Pattern Recognition (CVPR), jun 2015, pp. 5556-5565.

[22] T. Whelan, S. Leutenegger, R. F. Salas-Moreno, B. Glocker, and A. J. Davison, "ElasticFusion: Dense SLAM Without A Pose Graph," Robotics: Science and Systems, 2015.

[23] R. A. Newcombe, S. Izadi, O. Hilliges, D. Molyneaux, D. Kim, A. J. Davison, P. Kohi, J. Shotton, S. Hodges, and A. Fitzgibbon, "KinectFusion: Real-time dense surface mapping and tracking," in Mixed and augmented reality (ISMAR), 2011 10th IEEE international symposium on. IEEE, 2011, pp. 127-136.

[24] R. A. Newcombe, S. J. Lovegrove, and A. J. Davison, “DTAM: Dense tracking and mapping in real-time," in Computer Vision (ICCV), 2011 IEEE International Conference on. IEEE, 2011, pp. 2320-2327.

[25] L. Magerand and A. D. Bue, "Revisiting Projective Structure for Motion: A Robust and Efficient Incremental Solution," IEEE Transactions on Pattern Analysis and Machine Intelligence, p. 1, 2018.

[26] M. W. Tao, P. P. Srinivasan, S. Hadap, S. Rusinkiewicz, J. Malik, and R. Ramamoorthi, "Shape Estimation from Shading, Defocus, and Correspondence Using Light-Field Angular Coherence," IEEE Transactions on Pattern Analysis and Machine Intelligence, vol. 39, no. 3, pp. 546-560, mar 2017.

[27] J. Liao, B. Buchholz, J.-M. Thiery, P. Bauszat, and E. Eisemann, "Indoor scene reconstruction using near-light photometric stereo," IEEE Transactions on Image Processing, vol. 26, no. 3, pp. 1089-1101, 2017.

[28] S. Tijmons, G. C. H. E. de Croon, B. D. W. Remes, C. D. Wagter, and M. Mulder, "Obstacle Avoidance Strategy using Onboard Stereo Vision on a Flapping Wing MAV," IEEE Transactions on Robotics, vol. 33, no. 4, pp. 858-874, aug 2017.

[29] S. Yang, S. A. Scherer, X. Yi, and A. Zell, "Multi-camera visual SLAM for autonomous navigation of micro aerial vehicles," Robotics and Autonomous Systems, vol. 93, pp. 116-134, 2017.

[30] F. Liu, C. Shen, G. Lin, and I. Reid, "Learning depth from single monocular images using deep convolutional neural fields," IEEE Transactions on pattern analysis and machine intelligence, vol. 38, no. 10, pp. 2024-2039, 2015.

[31] E. Rublee, V. Rabaud, K. Konolige, and G. Bradski, "ORB: An efficient alternative to SIFT or SURF," in Proc. ICCV, vol. 58, no. 11. IEEE, 2011, pp. 2564-2571.

[32] V. Lepetit, F. Moreno-Noguer, and P. Fua, "Epnp: An accurate o (n) solution to the pnp problem," International journal of computer vision, vol. 81, no. 2, pp. 155-166, 2009.

[33] C. Kerl, J. Sturm, and D. Cremers, "Dense visual SLAM for RGBD cameras," in 2013 IEEE/RSJ International Conference on Intelligent Robots and Systems, nov 2013, pp. 2100-2106.

[34] F. Endres, J. Hess, N. Engelhard, J. Sturm, D. Cremers, and W. Burgard, "An evaluation of the RGB-D SLAM system," in Robotics and Automation (ICRA), 2012 IEEE International Conference on. IEEE 2012, pp. 1691-1696.

[35] J. Stückler and S. Behnke, "Multi-resolution surfel maps for efficient dense 3D modeling and tracking," Journal of Visual Communication and Image Representation, vol. 25, no. 1, pp. 137-147, 2014.

[36] T. Whelan, H. Johannsson, M. Kaess, J. J. Leonard, and J. McDonald, "Robust real-time visual odometry for dense RGB-D mapping," in 2013 IEEE International Conference on Robotics and Automation, may 2013, pp. 5724-5731.

[37] A. Handa, T. Whelan, J. McDonald, and A. J. Davison, "A benchmark for RGB-D visual odometry, 3D reconstruction and SLAM," in 2014 IEEE International Conference on Robotics and Automation (ICRA), may 2014, pp. 1524-1531. 